\title{
Future competencies for digitally aligned specialties: coping intelligently with global challenges
}

\section{Elena Libin}

Libin Coping Intelligence Institute, Chevy Chase, DC, USA, Advanced Studies in Coping Intelligence and Future Competencies Program at the Center for Advanced Research in Integrated and Digital Humanities (CARIDH), Russian State University for the Humanities, Moscow, Russia.

\begin{abstract}
The main goal of any education is to prepare students for future professional and life challenges. What is missing, however, from current curricula is the subject that deals with developing core competencies that are cross-cutting and focused on building the skills necessary for any specialties technological, medical or humanities. Main results from presented joined projects - Robotic Psychology \& Robotherapy Study, and the Coping Intelligence Project - build a configuration for a shared knowledge databank on human-technology interface, as well as on how coping intelligence impact academic achievements, professional expertise and life success. Evidence suggests that generalized efficient and inefficient problem solving in college students majoring in science, CS \& IT, and mathematics is associated with various profiles that differ by learning experiences with STEM disciplines, academic locus of control, and the level of academic achievements. Furthermore, implementing a coping intelligence approach in academic curricula elucidates the transformative role of core competencies, required for the successful management of risks and challenges associated with a variety of digitally aligned professional activities.
\end{abstract}

Keywords: Coping intelligence; core life competencies; digitally aligned specialties; global challenges; robotic psychology; robotherapy. 


\section{Introduction: bridging the innovation gap}

The main goal of any education is to prepare students for future professional and life challenges. What is missing, however, from current curricula is the subject that deals with developing core competencies that are cross-cutting and focused on building the skills necessary for any specialties - technological, medical or humanities.

The core of educational innovations for computer sciences (CS) \& information technology (IT), and other digitally aligned professionals, is grounded not in the computer-related disciplines itself, but rather in the set of abilities that often called "soft skills" or "integrated disciplines", or "cross-cutting and overarching competencies".

In our methodology of studying core competencies we merged conceptually and experimentally two different accounts. The one that takes technology as a starting point, namely Robotic Psychology and Robotherapy Project (Libin,A. \& Libin,E., 2004), and a human-centered approach focused on the vital role of Coping Intelligence in mastering both technological and psychosocial skills while dealing with the complexities of global challenges (Libin E.,2003).

The results suggest that an attempt to replicate a broad repertoire of interactive strategies, offered by a human coping intelligence with the programmed, AI-based, responses, is doomed by default. Even the most advanced innovative technology can not compensate for the lack of core human ability to cope with real world situations.

\section{Framework for exploring core life competencies for human-technology interactions}

Approach-I. Although we interact with digital gadgets daily, humans do not completely understand how the technology works. Moreover, we tend to use our imagination to transform tools into "living creatures", "partners", and even "friends". The goal of our Robotic Psychology and Robotherapy Project, a longitudinal study across countries and ages and abilities (2001 - present), is to explore what is real in our interactions with the engaging machines, called robots. We define robotic psychology and robotherapy as interdisciplinary fields of research and practice that focus on the compatibility between people and artificial creatures on different levels: sensory-motor, emotional, cognitive, and social. Robotic psychology studies the psychological significance of robots' behavior and its intertwining with elements of physical and social environments. Different classes of robots are aimed at fulfilling different human needs. Artificial creatures have their own distinct individuality, which manifests itself in the robot's design and behavioral configuration, in the same way that people and other living beings differ from each other by various parameters, such as weight and height, behavioral reactions and character, emotions 
and cognition, abilities and coping strategies. Analyses of person-robot interactions account for the essentials of both humans' and their artificial partners' behavior.

Methods-I. A cross-cultural mixed-methods study on children, youth, and elderly interacting with social communication robot (see Figure 1), characterized by advanced artificial intelligence and synthetic sensory feedback. More than 300 participants of different age, gender, competencies, and life experiences in USA, Russia, Ukraine and Japan were involved since 2001 with our project, funded by the Libin Coping Intelligence Institute, LLC (USA).
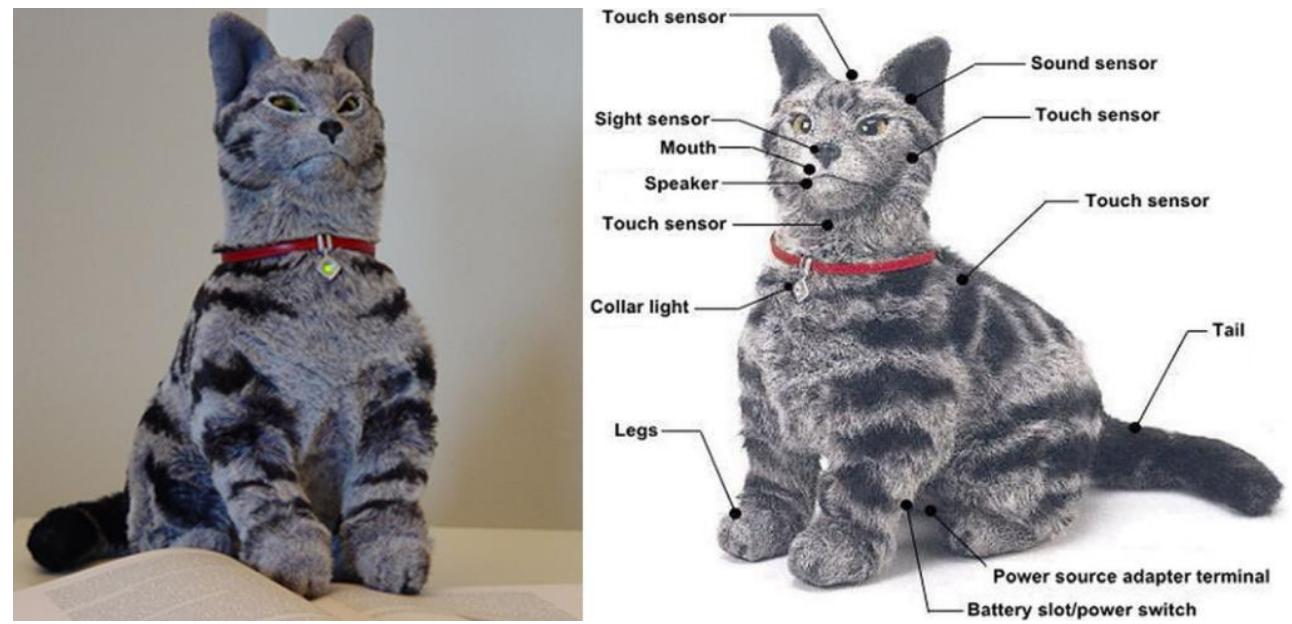

Figure 1. Robotic communication partner as a tool for RoboticPsychology and Robotherapy Project Source: Libin \& Libin, 2004.

Main findings-I. AI algorithms responsible for robot's behavioral configurations, despite their programming complexity, were extremely limited in their capacity to adapt to the needs of interacting humans challenged by the complexity of real-life situations. Human communication with the robot, as well as any interactions with the AI-based systems by that matter, are shaped not so much by the programming codes, but by our own life skills amplified by personal experiences and world-views.

In fact, the very way we deal with so called "smart" gadgets, characterized by any level of complexity, serves as a litmus test, or, to be more specific, a projective psychological assessment, thus revealing coping or destructive strategies in managing life difficulties.

\subsection{Coping Intelligence competencies in the era of global Artificial Intelligence}

Approach-II. As intriguing as the concept of AI sounds, human intelligence is a real blackbox with an unknown input and even more fascinating outputs when it comes to predicting the outcomes of our dealing with the complex real life situations. Our joined 
with the person-robot communication project is focused on the role of coping intelligence in managing the uncertainties presented by both modern digitalization and societal global challenges. The approach originated in 1995 and continued through present, aims to bridge scientific knowledge regarding efficient coping versus inefficient defense with real-life individual strategies for handling daily problems, including adequate decision-making and the intelligent use of emotions and smart behaviors in difficult circumstances. Coping processes, whether emotional, cognitive, or behavioral, are organized as a complex system defined by the result, evident from the successful resolution of life difficulties.

Coping Intelligence is defined by a broad repertoire of life skills essential for successful management of everyday challenges in order to sustain individual and social well-being (Libin, 2003, 2019). A concept of Artificial Intelligence (AI) evolves form the notion put forward by Marvin Minsky (1959), who defined AI as "the science of making machines do things that would require intelligence if done by men." We investigate decision-making in the real-life context, focusing on the criteria that differentiate decisions based on human intellect from algorithms programmed into AI.

Methods-II. Coping intelligence measurements were developed through a three-phase algorithm. As it was reported in previous studies (Libin, 2017), consequential steps or phases were implemented in designing both the quantitative and qualitative parameters of the Libin Coping Intelligence ${ }^{\mathrm{TM}}$ Questionnaire (LCIQ ${ }^{\circledR} 1.0$ ) including: a literature analysis and the pool of items development (Phase I), a content validity study of the questionnaire items and relevant global rating scales statements through the expert review panel (Phase II), and an exploration of the psychometric properties of the LCIQ ${ }^{\circledR} 1.0$ via factor analysis and Cronbach's alpha (Cronbach, 1951) reliability test (Phase III).

More then 450 people, mostly students of different specialties and professionals with various level of expertise, residing in Russia, USA, and Ukraine, were involved in the reported phase of the Coping Intelligence Project.

Main findings-II. Research focused on the assessment of effective and ineffective ways of handling daily challenges greatly improves our understanding of the close relationships between coping versus defense processes, as they define our interactions with the digital world. The proposed conceptual model, underlying the Libin Coping Intelligence ${ }^{\mathrm{TM}}$ Questionnaire (LCIQ ${ }^{\circledR} 1.0$ ), identifies the result as the primary factor that organizes any coping activity, whether emotional, cognitive, or behavioral, within the complex coping intelligence system. Depending on the result, any given coping activity can be estimated as efficient or inefficient, successful or unsuccessful. Thus, the functional systemic organization of coping efforts applied toward the resolution, or the result, is identified as the primary cross-cutting parameter of coping intelligence. The functional organization of coping efforts applied toward a resolution, as the primary cross-cutting parameter, 
differentiates between efficient and inefficient strategies. Successful efficient strategies are focused on resolving the difficulties in order to reach meaningful goals; while, inefficient defensive strategies diverge from resolving life difficulties (see Figure 2).

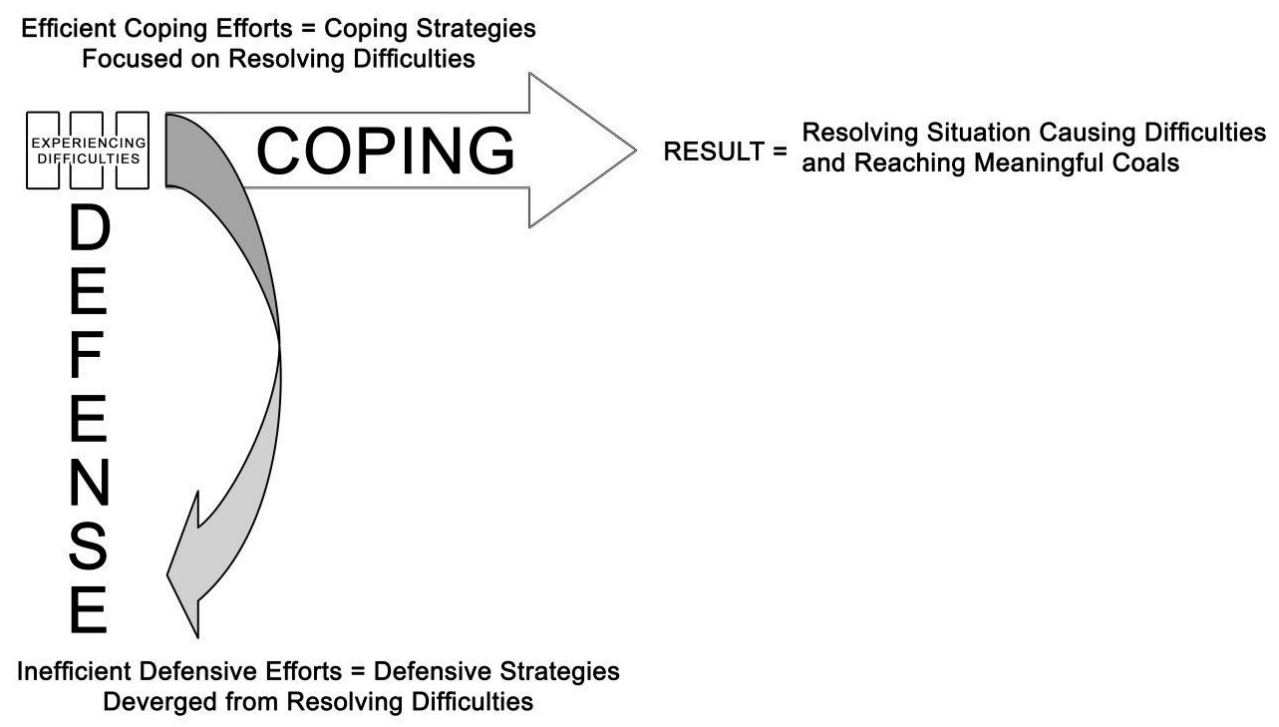

Figure 2. Efficient coping versus inefficient defensive strategies pathway. Source: Libin, E.2003.

Such a distinctive differentiation between two modes of human interaction with the physical and social environment triggers the enormous complexity of coping intelligence as a chief navigation system guiding us through life's hurdles.

\subsection{Future competencies for digitally aligned specialties}

Main results from joined projects build a configuration for a shared knowledge databank on how coping intelligence impact academic achievements, professional expertise and life success. There is plethora of evidence suggesting that generalized efficient and inefficient problem solving in college students majoring in science, CS \& IT, and mathematics is associated with various profiles that differ by learning experiences with STEM disciplines, level of anxiety, academic locus of control, and the level of academic achievements (Daniel, 2018).

Furthermore, implementing a coping intelligence approach in academic curricula allows the inclusive design of special educational programs aimed at the development of efficient coping skills that are vitally important in all areas of life.

Overall, individuals with deficient coping intelligence are characterized by patterns of defensive inefficient strategies, such as an inability to manage social relationships (Nicolas 
et al., 2013), a tendency to ignore the interests of others in favor of egotistic personal goals associated with bullying (Washington, 2015), and a propensity to be uncooperative and emotionally discouraging with oneself and others (Granieri et al., 2017).

Coping intelligence curriculum is of vital importance to digitally aligned professionals who develops technologies that shape our daily life. Indavertendly, today's students facing the same dilemma that is embraced by the society in general. Since the dawn of time the human kind mastered the set of skills necessary to survive and strive. The basic values remained the same - improving the quality of life, developing intellectual capacity, building relationships to achieve success and happiness. Artificial intelligence and digital innovations while remaining an important subject for higher education is burdened with the potential conflict between technological tools development and human skills to handle it (see Figure 3).
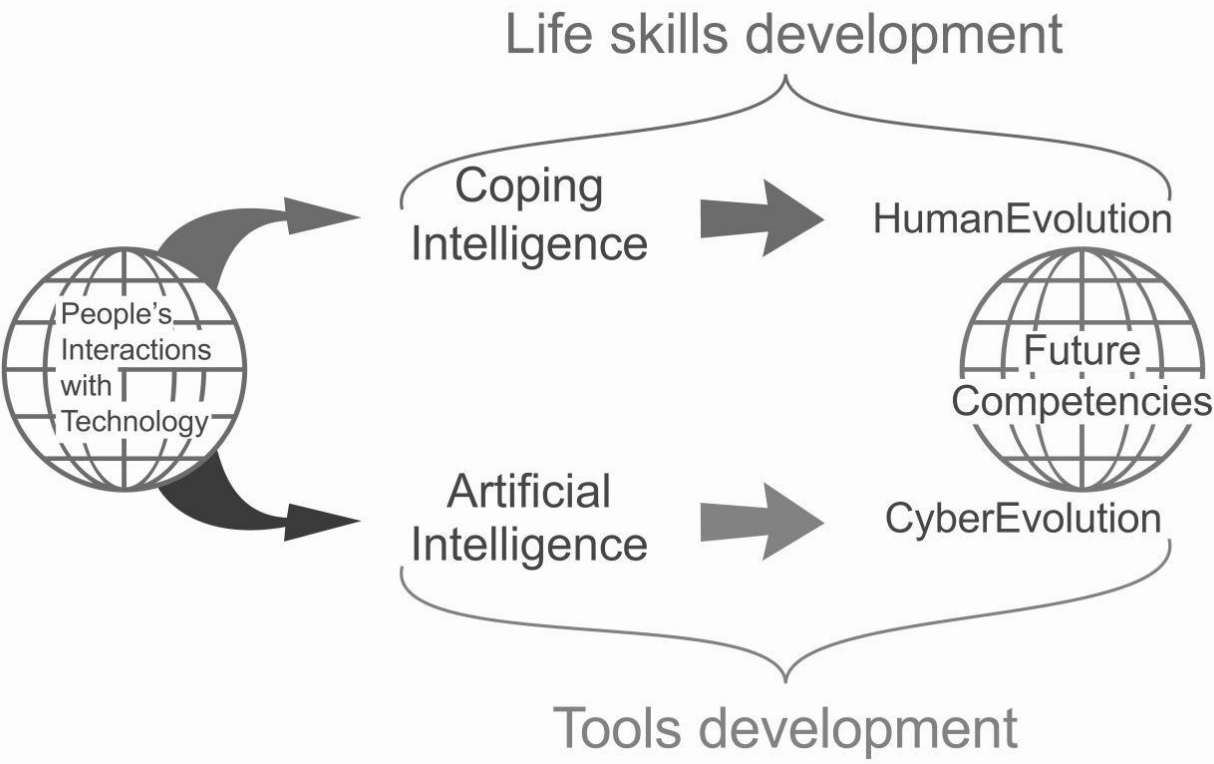

Figure 3. An innovation gap that needs to be addressed through the core competencies curricula.

Dangerous situations have been already documented when humans lost control of even guided artificial intelligence (Nicas et al., 2019). It is unknown what goals will artificial intelligence pursue running amok. But it is easy to predict, that learning competencies and professional expertise, enhanced with human coping intelligence, can balance even the most daring outcomes of technological expansion.

Being part of the design for advanced prospective studies, the coping intelligence approach elucidates the transforming role of coping competencies, required, along with the core soft 
skills, for the successful management of risks and challenges associated with a variety of professional activities. It is hard to overestimate the importance of effective coping skills in the context of the increasing complexity of modern life. A broad efficient coping repertoire helps each of us to successfully overcome obstacles triggered by socioeconomic pitfalls and obscurities of emerging technologies, and triumph over life adversity.

Acknowledgements. The author would like to express sincere gratitude to Professor Alexander V. Libin at Georgetown University, a Scientific Director at the Research Design \& Biostatistics Core, Georgetown-Howard Universities Center for Clinical \& Translational Science (Washington, DC, USA), for his valuable contributions to the article and, more specifically, for partnering over the years on both presented approaches.

\section{References}

Daniel, K.L. (2018). Towards a Framework for Representational Competence in Science Education. Volume 11 of Models and Modeling in Science Education, Springer, 277 p.

Granieri, A., La Marca, L., Mannino, G., Giunta, S., Guglielmucci, F., Schimmenti, A. (2017). The Relationship between Defense Patterns and DSM-5 Maladaptive Personality Domains. Frontiers in Psychology, 8, 19-26. doi:10.3389/fpsyg.2017.01926

Jack Nicas,J., Kitroeff,N., Gelles,D, and Glanz,J. (2019). Boeing Built Deadly Assumptions Into 737 Max, Blind to a Late Design Change. The New York Times, June 1. Retrieved on January 20, 2020: https: //www.nytimes.com/2019/06/01/business/boeing-737-maxcrash.html

Libin A. \& Libin, E. (2004). Person - Robot Interactions From the Robopsychologists Point of View: The Robotic Psychology and Robotherapy Approach. Invited Paper. Proceedings of the IEEE, 92 (11), 1789-1803.

Libin, E. (2003). Individual Differences in Efficient and Inefficient Coping Strategies. Ph.D. Thesis, Institute of Psychology at the Russian Academy of Education.

Libin, E. (2017) Coping Intelligence: Efficient Life Stress Management. Front. Psychol. 8:302. doi: 10.3389/fpsyg.2017.00302

Libin, E. (2019). Coping Psychology. Moscow: URAIT, 360 p.

Minsky, M. (1959). Some Methods of Heuristic Programming and Artificial Intelligence. In: Proc. Symposium. on Mechanization of Thought Processes, vol 1. 5-27. D.V. Blake and A.M. Uttley (eds.), Natl. Physical Lab., Teddington, England, HMSO, London.

Nicolas,V., Mjeldheim,G.S., Weiss,K. \& Yusupova,A. (2013). Mars-105 study: Timecourses and relationships between coping, defense mechanisms, emotions and depression. Journal of Environmental Psychology 35, 52-58.

Washington, E. (2015). An Overview of Cyberbullying in Higher Education. Adult Learning. 26. 21-27. doi:10.1177/1045159514558412. 